\title{
Guidelines to Overcome the Electronic Health Records Barriers in Developing Countries
}

\author{
Hussein Boshnak \\ Faculty of Medicine \\ Ain Shams University, \\ Cairo, Egypt
}

\author{
Sayed Abdel Gaber \\ Faculty of Computers and \\ Information \\ Helwan University, Cairo, \\ Egypt
}

\author{
Amany Abdo \\ Faculty of Computers and \\ Information, Helwan \\ University \\ Cairo, Egypt
}

\author{
Engy Yehia \\ Faculty of Commerce and \\ Business Administration \\ Helwan University, Cairo, \\ Egypt
}

\begin{abstract}
Conversion from paper-based medical records to electronic Health Records provides significant benefits to healthcare. However, there are some challenges perceived by healthcare providers when implementing Electronic Health Records. This paper aims to present some guidelines to overcome barriers associated with implementing Electronic Health Records in developing countries. This paper also presents an overview of Electronic Health Records Barriers discussed in several studies and review some studies performed in developing countries to explore the implementation barriers of the Electronic Health Records.
\end{abstract}

\section{General Terms}

Electronic Health Records

\section{Keywords}

Electronic Health Records, EHR Barriers, Developing Countries.

\section{INTRODUCTION}

Electronic Health Records [1] is a longitudinal health record with entries by healthcare practitioners in multiple sites where care is provided [2]. Electronic Health Records have been developed and implemented by some institutions and countries while others are currently planning to develop it. EHRs have been developed in different forms and types, and what one country calls an EHR may not be the same as that developed in another country [2]. Conversion from paper medical records to electronic medical records provides significant clinical and operational benefits [3]. The potential benefits of EHR including improve efficiency and effectiveness of healthcare delivery systems, improve patient safety and reduce medical errors, enhance patient's engagement with their health care by providing patients with an electronic copy of their health information upon request, enable clinical decision support by suggesting, from the patient data, a diagnosis or a therapeutic strategy. EHR can improve the administrative processes including patient care delivery, care management, financial and patient selfmanagement, reduce the health care costs by reducing incidences of needless duplicate or repeat tests and procedures currently conducted by health care professionals, Allow realtime sharing of patient information amongst health care providers such as physicians, physiotherapists, imaging professionals and pharmacists, as well as between providers and patients. EHR enhance the ability to integrate information from multiple sources to make evidence-based decisions and enhance the ability to communicate information and findings to other providers and patients. EHR offer tremendous potential as a research tool and provide a rich, diverse data set that is an invaluable source of information for health researchers [3-7]. EHR is not a single system, it is a collection of inter-locking systems that were tied to a series of complex clinical and administrative workflows [3]. A successful transition from paper-based to electronic health records in the healthcare organizations requires careful management and coordination of many aspects [8]. Despite the many benefits of having EHRs, there are still several barriers when systems are first developed, especially in developing countries [9]. Challenges with EHRs implementations in developing countries including the availability of resources, such as electricity and internet, inadequate infrastructure, the location of the care center, physicians resistance to the new technology, lack of information technology skills, poor strategies and policies, as well as the financial costs of implementing and maintaining EHRs [9, 10]. Developing countries interested in EHR must fully aware of the expected advantages and must analyze each of the choices to ensure the whole practice workflow is considered. Developing countries also must be fully understand the barriers and challenges associated with adoption and implementation of EHRs and how to overcome them [3, 8]. This research discusses the barriers associated with implementing EHRs in developing countries and aims to recommend some guidelines to overcome these barriers. Section 2 presents the EHRs barriers, section 3 introduces some studies performed in developing countries to explore the implementation barriers of the EHRs, and section 3 provides some guidelines to overcome these barriers

\section{ELECTRONIC HEALTH RECORDS BARRIERS}

The EHR has the potential to address many of the current challenges of healthcare paper-based systems, and to improve of efficiency, effectiveness of healthcare delivery systems, and patient safety [11]. Despite the advantages of Electronic Health Records, the development rate of these systems is still low and meets challenges which make physicians and healthcare professionals resist to develop them [12]. Healthcare executives are facing significant barriers to EHR implementation. In this section, an overview of these barriers collected from several studies will be discussed.

\subsection{People Barriers}

Among the various health care professionals who use different components of the EHR are physicians, nurses, patients and other stockholders. There were several studies reported the most important perceived barriers among primary care physicians, patients, nurses and other stakeholders [4, 13-15]. Physicians have concerns about the use of EHRs based on their personal thoughts and issues, knowledge, and perceptions $[11,14,16-26]$. Major concerns among Physicians including disruption of clinical workflow resulting in loss of efficiency and productivity, increased physician workload and physician resistance, negative impact on 
interactions between physicians and patients such as loss of eye contact and loss of communication with the patient. Physicians although concerns about usability and flexibility issues such as slow EHR system and inability to type quickly enough, writing long prose notes and reported that using the computer in front of the patient was considered unprofessional and time-consuming. Physicians think that using EHRs will take more time for each patient than using paper as, in some situations; it might be more convenient for physicians to use paper records during the patient session. Other concerns including financial costs associated with implementing and maintaining the EHRs Lack of adequate IT resources, implementation complications and lack of standardization and interoperability, unable to select an EHR system that meets their specific requirements. Concerns regarding system downtime and a lack of understanding of benefits resulting from EHR implementation, acceptance and Lack of belief in EHRs, training to use her system and concerns about the loss of their control of patient information and working processes since these data will be shared with and assessed by others.

\subsection{Financial Barriers}

Several surveys report that the monetary aspect is one of the major barriers of adopting EHRs [25]. Cost has greatly limited the adoption of EMR systems, particularly in small medical practices.[17]. The most common organizational concern reported was around financial costs associated with all stages of the EHR implementation such as planning, consulting services, contracting, start-up, purchasing of hardware and software, and ongoing costs for training and maintenance. financial barriers can be categorised as: Start-up and Contracting costs, Ongoing and Maintenance costs, Lack of financial resources, and Uncertainty over Return on Investment (ROI) $[13,14,20,25,27-30]$. Start-up costs include all the expenditure needed to get an EMR system working in the physician's practice, such as the purchase of hardware and software, selecting and contracting costs and installation expenses. Many researchers state that these costs are significant and therefore should be regarded as a high barrier to physicians adopting EMRs, especially for those without large IT budgets [14]. Ongoing and maintenance costs include the long-term expenditures incurred in monitoring, modifying, upgrading and maintaining EHRs, which will be significant [14]. Administrative and clinical executives at health care facilities cite the lack of financial support as the biggest barrier to implementing information technology [13]. Physicians worry that their practices will face substantial financial risks and that it could take years before they see a return on the investment [14].

\subsection{Data Structure and Records Barriers}

Transforming patient's records from paper-based to electronicbased system is a commonly barrier to the adoption of the EHR systems. Data Structure and records barriers can be classified to some barriers such as: Difficulties in incorporating external data, Time required for data migration, Storing of Unstructured data in to the EHR Data structure, Long-term storage and protection of records, Synchronization of records, and Inadequate standards for data representation and vocabulary [13, 14, 20, 28, 29, 31-33]. Implementing an EHR means switching from paper-based to electronic based systems, and this involves transferring records between the two systems. Some organizations scan the documents and retain them as images. However, physicians are less satisfied with these scanned documents because they are hard to read [14]. Clinical data is represented in structured and unstructured form. Unstructured clinical data must be transformed into structured data if health information professionals want to analyze the data and use it to improve care $[33,34]$. A lack of industry-wide standards means that integrating disparate clinical systems, exchanging patient information and collaborating among entities is complicated if not impossible [13]. Lack of universal standards for vocabulary and data representation poses a major impediment to data sharing and interoperability. Multiple vocabularies exist across health care entities, complicating the exchange of health information between providers[28].

\subsection{Technological Barriers}

Technological barriers present a major roadblock to the implementation and adoption of EHR technologies. Technological barriers consist of Hardware limitations, System limitations, System Complexity, Lack of System customizability, Required infrastructure and Interconnectivity, Lake of data Standardization, Lake of system Interoperability, and Lack of Reliability [4, 11, 13, 14, 19, 20, 25, 27, 28, 30, $35,36]$. Some researchers state that some practices lack these 'basic' facilities/hardware needed to support EHR implementation and that this issue blocks the widespread adoption of EHRs [14]. The complexity and usability problem associated with EHRs results in physicians having to allocate time and effort if they are to master them. It is also possible that a lack of skills leads the physicians to regard the EHR system as extremely complicated [14]. Some systems failed to meet expectations, necessitating considerable system modification to establish required functionalities; in some instances the expected functionality has not yet been delivered[28]. Many surveys show that one reason why physicians do not adopt EHRs is that they cannot find a system that meets their special needs[14]. For some of these technologies, the infrastructure to support the application requires significant coordination among competing health care entities [13]. The challenges associated with using noninteroperable EHR systems such as inadequate electronic data exchange may negatively impact workflow and productivity, which in turn contribute to clinicians' resistance to adopt these systems [20].

\subsection{System Development Barriers}

Planning, Selection, development and implementation of EHR system are considered as important factors in the adoption of EHR systems. System development barriers consist of preimplementation phase barriers, implementation phase barriers, training issues, and post-implementation phase barriers [20, 37]. There is a concern that the organisation will be unable to select an EHR system that meets the needs of physicians due to an overwhelming number of EHR vendors [20]. There are some of factors that have adversely impacts in the project preimplementation phase include: Insufficient communication to end users and stakeholders regarding the project goals and objectives, Development of an inappropriate and unrealistic project timeline, Lack of project manager participation in preproject, Inadequate project workplan development, unrealistic project scope, Inadequate amount of budgeted capital for projected project costs. One of the main complaints among IT project managers is the lack participation from end users and stakeholders during the project implementation process. Postimplementation phase are often high stress phase specially with worried end-users and an almost imperious feeling of vulnerability and uncertainty that is shared between all parties $[20,37]$. 


\subsection{Organizational Barriers}

The organizational characteristics of individual and Physicians work in medical and hospitals practices will be a factor in the adoption of EHRs [14]. Successful EHR implementation also requires significant operational change in organizational culture, structure and workflow. Organisational barriers could be related to Organizational size, Organizational tybe, Organizational Culture, Resistance to Organizational Change (Inertia), and Redesign of Office Work Flow [14, 30, 34]. Several Surveys show that physicians in larger medical practices have a higher HER adoption rate than those in smaller practices. It was also found that "physicians in larger practices are more likely to use available functions in their EMRs than those with EHRs in smaller practices. According to several studies, physicians who are contracted to or employed by a medical practice are more likely to use EHRs than those who work in their own practices because of the high start-up and ongoing costs [14]. While there are a number of technical and resource challenges to EHR implementation, culture change has by far been the most challenging aspect of implementing new EHR system. The existing culture of the organization may present barriers throughout the implementation process between team work, particularly if there is lack of trust and cooperation [30]. Despite of the positive effects of the EMRs usage in medical practices, the adoption rate of such systems is still low and meets resistance from physicians [29]. The process of using EHRs effectively requires a different set of actions than using paper records, and redesigning workflow is recommended as the first step towards successful EHR implementation [5].

\subsection{Managerial Barriers}

The organization management plays a vital role in implementing the EHR system but there were some managerial barriers that influence the EHR adoptions. Such as, Project resource management, Lack of leadership, and Lack of policies on key issues [4, 13, 14, 37]. Without the presence of a champion, an individual who is familiar with the system and can help others learn the system, it is easier for those involved in implementation and adoption of systems to become discouraged and use the system less [4]. The Leadership, board of directors and senior management must believe that technology is the key for the future. Without public support on how privacy will be addressed, EHR systems will not be able to proceed [13].

\subsection{Legal Barriers}

Security and privacy concerns are one of the most discussed legal barriers to the implementation and adoption of an interoperable EHR system [4]. The consequent inadequate disclosure of sensitive patient information might lead to legal problems [14, 25, 30, 38, 39]. Physicians doubt whether EHRs are a secure store for patients' information and records, and fear that data in the system may be accessible to those who are not authorized to obtain it [14]. Some of the broad privacy issues relating to including What information should be included in the EHR?, Who should have access to the EHR?, How will a patient be able to access his or her own EHR?, and In what instances can the information in an EHR be used for secondary purposes (e.g. research, administration)? [13].

\subsection{Social Barriers}

Physicians in medical practices work together and communicate with other parties in the healthcare industry, such as patients, insurance companies, vendors, administrative staff, subsidizers, and managers. The decision-making process using EHRs by physicians is influenced by these parties and will also affect the relationship between patients and physicians. Social barriers include Lack of support from other colleagues, Uncertainty about the vendor, Lack of support from external parties, and Lack of support from the management level [14]. The complaints about workload increases, the lack of technical skills, negative perceptions and resistance to using EHRs leads to a lack of support from these colleagues, which impedes physicians in further adopting the system. Physicians are unwilling to enter this market without trust in trustworthy and reputable vendors and their decision making was affected by regional and local organizations that were not active in the EHR discussion [14].

\section{EHR ADOPTION IN DEVELOPING COUNTRIES}

Due to the EHRs implementation scenarios that exist in developing countries, the adaptation of EHR systems is limited and has several challenges[40]. Several studies performed on the developing countries hospitals to explore users and physicians concerns of adopting EHR system. Some of these studies will be reviewed in this section.

Sana Z. Khan, et al. (2012) performed a qualitative study at two Bangladeshi hospitals to articulate and analyze users' expectations hopes and fears regarding the use of EHR systems. Sana Z. Khan et al. performed this study to explore how to implement EHRs in developing countries by considering fears and concerns of users when initiating EHRs. Sana Z. Khan et al study builds on data from two hospitals, government hospital and non-government hospital. Data was collected using a combination of interviews and observations using a technology the Unified Theory of Acceptance and Use of Technology (UTAUT) acceptance model to investigate healthcare staff's adoption of health information systems and capture the physicians' hopes and fears regarding the use of EHR. Despite the authors found that there were many hopes of EHR systems to be convenient, time saving, productive, efficient, and to support them on their professional decision making, many more fears were articled. These fears were related to resistance of change which physicians prefer the manual system rather than using a computer screen. Time consumption is other fear of physicians, they thought that using an EHR would negatively influence the time they could spend with patients. Other fears related to lack of computer skills and training, lack of computer trusting to record patient data, need of funds, technical insufficiency and need for rules and policy for adopting EHR system [10].

Khwima E., et al (2017) performed a study aims to investigate factors that affect the utilization of EMR systems by health workers at central hospitals in Malawi. In this study, health workers were interviewed, using a semi structured questionnaire where EMRs were first introduced in the country. The authors applied both quantitative and qualitative techniques. Health workers investigated in this study included clinicians, laboratory technicians, data clerks, nurses, and HIV counsellors. The results showed positive attitude towards EMR usage, health workers found EMRs to be quicker, more accurate, more secure and reduce healthcare staff workload when comparing to paper records. However, the health workers have many concerns that negatively affected EMRs using including the lack of EMR training, lack of managerial support, the lack of hardware infrastructure and connectivity problems [41].

Nader Shams Amiri (2012) performed a study for assessing the situation of EHR in Iran. The author used observation and semi-structured interviews to investigate the EHR concerns of 
users in Iranian hospitals. The results showed that common issues reported from the interviews including the cost of implementing EHR system, lack of financial resources, lack of standards and interoperability, legal restrictions and confidentiality problems for sharing information. Also, some barriers reported from the observations including resistance to using EHR from some users, low speed for sharing information between users, lack of structured procedures, and lack of using all EHR capabilities [42].

Abdullah Alkraiji et al. (2011) performed a study aims to investigate the factors influencing the adoption process of HIT-related standards in Saudi Arabia. The authors collected the data using unstructured interview, semi-interviews and documentation. The authors have identified many factors which have an impact on the adoption process of HIT-related standards. These factors are as follows: data standards, integration and interoperability, organization characteristics, policy and procedure, organization readiness, clinician engagement, the direct and indirect cost, and external support [43].

AS Eldin et al. (2013) presented a study to assess the state of EHRs in Egypt by surveying a sample of public, private, and educational hospitals. The authors collected the data using questionnaire. The results showed that there were many barriers that prevented the EHR system adoption. These barriers including lack of computer skills, access technical support, start-up financial costs, ongoing financial costs, lack of uniform standards, technical limitations, privacy and security concerns, and training and productivity loss [44].

Jessica Stadelmann (2012) performed a study to evaluate and analyze the challenges and weaknesses for implementation of E-Health in Cairo University Hospitals in Egypt. This study used the California Community Clinics EHR Assessment and Readiness survey. Jessica Stadelmann used interviews and survey in order to gather information on the system in Cairo University Hospitals. The main key issues were identified including lack of communication and governance, finance and budget problems, and quality and operational performance [45].

\section{GUIDELINES TO OVERCOME EHR BARRIERS}

This section describes the procedures and guidelines necessary for overcoming most perceived barriers.

To overcome time constrains issues

- Adapt EHR system to current medical practice and workflow activities [14].

- $\quad$ Provide the EHR systems with simple interfaces and forms to save the physician time and effort while entering patient visit notes [46].

- Address workstation availability and system speed issues by healthcare organizations to minimize time required by physicians to document patient information [20].

- Ensure strategic placement of the computer workstations for effective using of clinical reminders and communication between physician and patient during patient encounter [20]

To overcome Training issues

- Improve data processing analysis, data collection instruments, and decision-making as a part of the national curriculum in schools of nursing and medicine [16].

- Organise refresher courses on the information system for all users levels [16].

- Educate the providers where to locate information, how to access different components of the chart, and how to document quickly all the required information elements during the encounter [24].

- Ensure that EHR users receive regularly scheduled training on the organization's procedures and policies for maintaining EHR integrity, including security and $\log$ in, authorship/authentication, validity of data, data transmittals, and use and storage of data [47].

- Ensure that documentation of the education programs are performed and become a part of the physician, provider, or employee's permanent medical staff or human resource record for the event of any possible future issues regarding false or fraudulent entries [47].

- To overcome data documentation issues

- Improve Data documentation rates through staff training and education [48].

- Enhance the EHR system with advanced technologies such as voice recognition and mobile devices to enter and access clinical data [11]

- Educate physicians and support ongoing training to avoid documentation errors [14].

- Design Policies to minimize recording of patient data available elsewhere in the EHR and discourage copying features as a way of improving clinician productivity [49].

- Implement a user friendly help function and help desk for the physicians [14].

- Provide the EHR systems with standardized templates, data collection forms, or patient record forms functionalities to assist in provider documentation [46].

- Monitor EHR documentation practices, such as copying and pasting text to ensure they are appropriate and apply corrective action if an inappropriate documentation practices is identified [49].

To overcome Poor user acceptance and participate barrier

- Consider the role of users in the implementation of health information technology in practices and healthcare institutions for a successful telemedicine implementation program [50].

- Work out a plan for cultivating positive change attitudes toward using the EHRs to prevent the challenge of the fear of the unknown by users [50].

- $\quad$ perform extensive software testing to avoid users' frustration with software problems which can 
quickly escalate to the entire EHR system and result in resistance to implementation [20]

- Formalize roles and responsibility in collaborating arena to ensure continuity of record keeping and enhance their participation and role in the user community in the development cycle of the EHRs [50].

- $\quad$ Engage EHR users with clinicians prior to the EHR design stage, to ensure that it is suitable for end users, and improve their understanding of the benefits resulting from EHR implementation [20].

To overcome usability issues

- Integrate the Usability and human factors approaches into the design of EHR system development in order to overcome the prevailing mismatch between clinical work and information technology to support practices and improve patient safety [26].

- Include the EHR usability in the EHR certification process and define what a vendor's product is allowed to do [49].

- Develop and implement procedures and policies by Healthcare organizations and other providers pertaining to appropriate EHR use [49].

- Provide the EHRs users with flexible and customizable system to address diverse needs without creating confusion and communication breakdown [11].

- Apply federal regulations to mandate that EHR system vendors employ usability standards that enhance system safety and information integrity [49].

To overcome data entry issue

- Verify validity of information on data entry by the EHR system functions to either warn or not allow impossible information, for example, entering a prostate examination for a female [47].

- $\quad$ Provide EHR systems with the ability of Checking for duplication to not allow duplication of patient identification numbers or codes and the ability of checking for conflicting to warn of conflicting medical management options, such as lifethreatening drug interactions [47].

- Establish internal reporting system to address errors and issues related to EHR system [49].

- Control and limit automatic creation of information whether through a copy-and-paste or pull-forward function, selection of generic documentation, or use of "auto-neg" or other documentation by exception functions to commit fraud, and they are also a source of "dirty data" that will affect on patient care [47].

- Monitor additions and corrections to the medical record that should always be made available to the user of the record (e.g., incorrect information was originally recorded about the patient) [47].
To overcome changing in workflow

- Analyse the clinical workflows early and continue through implementation to address risks that are foreseen as well as those that arise during use [11].

- Include the training and support during redesign of workflow to enhance effectiveness of future interventions. [48]

To overcome System Interface Design Issues

- Keep the design of the information system, Operational cards, forms, and registers as simple as possible [16].

- Select sensitive, valid, and specific indicators to increase data quality [16].

- Establish an industry standard for quality principles and processes for EHR design to adopt by EHR system developers [49].

- Minimize the number of levels in the EHRs during transfer and processing of data in order to avoid errors. [16].

- Involve users in the design of the system to help in choosing a best suited information system to the user and improve the quality of the data produced.[16].

- Involve Checking procedures by Supervisors in the information systems design phase as a cost-effective ways to improve data quality [16]

- Consider how the system delivers information to each group of users because pertinent information may vary across provider types and clinical environments [11].

To overcome the unstructured data issues

- enable reuse of unstructured data by using methods such as Natural Language Processing [51] to extract useful information from unstructured free-text [48, $52,53]$

- Use a Standardization of data definitions and structure for clinical components which may include the use of text expanders or smart text and limit the use of free text as much as possible [46].

To overcome lack of standards

- Support the EHR system with international communication and vocabulary standards like the Health Level Seven electronic interchange standard and Systematized Nomenclature of Medicine Clinical Terms (SNOMED CT) to support information exchange through uniform and defined languages [11].

- Enhance the EHR systems with data dictionary that provide standard data field definitions for each data element to support and adopt more consistent use of data elements and terminology to improve the use of data in reporting [46].

- Provide the EHR systems with a standardized format functionality to ensure consistency. [46]. 
- Apply Federal regulations to establish monitoring processes and EHR system standards [49].

To overcome lack of interoperability

- Use of auto-format functionality or use of structured standardized data to enable the sharing and exchange of health information with HIEs and other organizations. For example, consider entering information, such as vital signs, as discrete data into correctly formatted fields, versus allowing free text entry into a field [46].

- Ensure that EHR system vendors support the free exchange of information and not prevent information sharing among EHR system users [49].

To overcome lack of up to date patient information

- $\quad$ Provide the EHR system with features that support maintain and up to date of patient information and identify how new information will be incorporated in a timely manner [11].

- Engage patients online and processing their feedback to assist in maintaining accurate and complete medical records and improve the accuracy of the information stored in their EHRs [54]

To overcome software limitations

- Select the appropriate software that must be able to accomplish the required clinical processes, interface with or replace existing hospital infrastructure, and not disrupt clinician workflow [11].

- Ensure that EHR systems not to be marketed without being approved to assess their safety and accuracy [49].

- Support the EHR system with well-established internal and external processes for addressing and reporting incidents and flaws events and developing solutions to them [11].

- Develop a plan for system testing to identify problems and workflow issues before implementation and during ongoing use so that they can be addressed before there is potential for patient harm [11].

To overcome hardware limitations

- Select an appropriate hardware that must be able to support software functions and facilitate clinician processes [11].

- Consider the followings during hardware selection: minimal use of space, electrical capacity, ability to document both at the bedside and outside the patient room, Flexibility, ease of use and the number of devices needed to accommodate peak staffing times [30].

- Provide technical support during implementation and a problem reporting system to document problems and receive prompt feedback [20]

- Include potential upgrades and hardware additions in the selection process and budget such as additional computer workstations to facilitate clinician access [11].
- $\quad$ support EHRs users with IT facilitators, such as flatscreen monitors on mobile arms, availability of convenient computers at numerous locations in the encounter office, in the hospital and at the homes of on-call physicians [20]

To overcome Security and Privacy barriers

- Improve the Authentication process by using complex and longer passwords and Changing them often to prevent unauthorized access [11].

- Enhance the security by using some form of multifactor authentication, that use more than one different method to identify a user, such as a password or personal identification number (PIN); smart card or a digital token ; and biometrics such as fingerprints or retinal scans [11].

- Improve authorization by assigning varied privileges to the users so that they can only see or use the parts of the chart that are relevant to them to protect patients from malicious breaches in confidentiality and prevent a well-intended clinician from inadvertently reading the wrong chart or writing an order on an incorrect patient [11].

- Protect personal health information by applying some form of encryption to make it unreadable to anyone without algorithm or specific key used to convert unreadable data to a readable form [11].

- Protect the network security from threats using Firewalls to monitor and restrict data communications and stop unauthorized transmissions of health information [11].

To overcome financial barriers

- Examine profitable examples from other EHRs implementations, provide documentation on return on investment, and provide financial compensation [14]

- Devote resources to provide ongoing hand-on support long after new technology is initially implemented, such as software updates, equipment upgrades and replacement, as well as ongoing maintenance in the budget when estimating implementation costs [30].

\section{CONCLUSIONS}

In this paper, some guidelines were presented to overcome implementation barriers of Electronic Health Records in developing countries. Electronic Health Records Barriers mentioned in several studies were discussed. This paper also reviewed some studies performed in developing countries to explore the implementation barriers of the Electronic Health Records. These guidelines should be considered by health care providers before and while developing Electronic Health Records.

\section{REFERENCES}

[1] Wanderer, J.P. and J.M. Ehrenfeld, Overview of Electronic Health Records, in Monitoring Technologies in Acute Care Environments. 2014, Springer. p. 379-384.

[2] Organization, W.H., Electronic health records: manual for developing countries. 2006: Manila: WHO Regional Office for the Western Pacific. 
[3] Hassibian, M.R.J.R.I.J.o.M., Electronic health records acceptance and implementation in developing countries: Challenges and barriers. 2013. 1(1): p. 6-11.

[4] Scott, J., Perceived Barriers to the Use of Electronic Health Records for Infectious Disease Surveillance in Canada. 2015, Université d'Ottawa/University of Ottawa.

[5] Kissam, S., et al., Barriers to meaningful use in Medicaid: analysis and recommendations. Rockville, MD: Agency for Healthcare Research and Quality, 2012.

[6] Cuggia, M., P. Avillach, and C. Daniel, Representation of patient data in health information systems and electronic health records, in Medical Informatics, eHealth. 2014, Springer. p. 65-89.

[7] Johnson, C., D. Johnston, and P. Crowle, EHR usability toolkit: A background report on usability and electronic health records. Rockville, MD: Agency for Healthcare Research and Quality, 2011.

[8] Kamal, J.I.A.J.D., Implementation of Electronic Medical Records in Developing Countries: Challenges \& Barriers. 2018. 7(3).

[9] Kalogriopoulos, N.A., et al. Electronic medical record systems for developing countries. in Engineering in Medicine and Biology Society, 2009. EMBC 2009. Annual International Conference of the IEEE. 2009. IEEE.

[10] Khan, S.Z., et al., Hopes and fears in implementation of electronic health records in Bangladesh. 2012. 54(1): p. $1-18$.

[11] McGinn, C.A., et al., Comparison of user groups' perspectives of barriers and facilitators to implementing electronic health records: a systematic review. BMC medicine, 2011. 9(1): p. 46

[12] Khalifa, M., Barriers to health information systems and electronic medical records implementation. A field study of Saudi Arabian hospitals. Procedia Computer Science, 2013. 21: p. 335-342.

[13] Vishwanath, A. and S.D. Scamurra, Barriers to the adoption of electronic health records: using concept mapping to develop a comprehensive empirical model. Health Informatics Journal, 2007. 13(2): p. 119-134.

[14] Boonstra, A. and M. Broekhuis, Barriers to the acceptance of electronic medical records by physicians from systematic review to taxonomy and interventions. BMC health services research, 2010. 10(1): p. 231.

[15] Kruse, C.S., et al., Barriers to Electronic Health Record Adoption: a Systematic Literature Review. Journal of medical systems, 2016. 40(12): p. 252.

[16] Lippeveld, T., R. Sauerborn, and C. Bodart, Design and implementation of health information systems. 2000: World Health Organization Geneva.

[17] Priebe, C.J. and E. Rose, Workflow automation with electronic medical records. Informatics in primary care, 2002: p. 152-165.

[18] Baron, R.J., et al., Electronic health records: just around the corner? Or over the cliff? Annals of internal medicine, 2005. 143(3): p. 222-226.

[19] Association, H.F.M., Overcoming barriers to electronic health record adoption. Results of survey and roundtable discussions conducted by the Healthcare Financial Management Association, 2006.

[20] Cotea, C., Electronic health record adoption: perceived barriers and facilitators. Research Coordination Unit, CMVH, 2010.

[21] Kim, M.S., et al. Usability Challenges and Barriers in EHR Training of Primary Care Resident Physicians. in International Conference on Digital Human Modeling and Applications in Health, Safety, Ergonomics and Risk Management. 2014. Springer.

[22] Courtney, K.L., A. Kuo, and O. Shabestari, Driving Quality in Informatics: Fulfilling the Promise. Vol. 208. 2015: IOS Press.

[23] Doberne, J.W., et al. Barriers to Information Access in Electronic Health Records during Initial Patient Visits A Qualitative Study. in Proceedings of the International Symposium on Human Factors and Ergonomics in Health Care. 2015. SAGE Publications

[24] Matthews, E.B., Integrating the Electronic Health Record into Behavioral Health Encounters: Strategies, Barriers, and Implications for Practice. Administration and Policy in Mental Health and Mental Health Services Research, 2015: p. 1-12

[25] Rahman, R. and C.K. Reddy, Electronic Health Records: A Survey. Healthcare Data Analytics, 2015. 36: p. 21

[26] Kaipio, J., et al., Usability problems do not heal by themselves: National survey on physicians' experiences with EHRs in Finland. International Journal of Medical Informatics, 2017. 97: p. 266-281.

[27] Thakkar, M. and D.C. Davis, Risks, barriers, and benefits of EHR systems: a comparative study based on size of hospital. Perspectives in Health Information Management/AHIMA, American Health Information Management Association, 2006. 3.

[28] Goroll, A.H., et al., Community-wide implementation of health information technology: the Massachusetts eHealth Collaborative experience. Journal of the American Medical Informatics Association, 2009. 16(1): p. 132-139.

[29] Ajami, S. and T. Bagheri-Tadi, Barriers for adopting electronic health records (EHRs) by physicians. Acta Informatica Medica, 2013. 21(2): p. 129.

[30] Jones, E. and F. Blavin, Lessons from the Literature on Electronic Health Record Implementation. 2013.

[31] Bushinak, H., S. AbdelGaber, and F.K. AlSharif, Recognizing The Electronic Medical Record Data From Unstructured Medical Data Using Visual Text Mining Techniques. International Journal of Computer Science and Information Security, 2011. 9(6): p. 25.

[32] Sittig, D.F. and H. Singh, Legal, ethical, and financial dilemmas in electronic health record adoption and use. Pediatrics, 2011. 127(4): p. e1042-e1047.

[33] Townsend, H., Natural Language Processing and Clinical Outcomes: The Promise and Progress of NLP for Improved Care. Journal of AHIMA, 2013. 84(2): p. 4445

[34] Ayatollahi, H., N. Mirani, and H. Haghani, Electronic health records: what are the most important barriers? 
Perspectives in health information management, 2014. 11(Fall).

[35] Kim Futrell, M., Structured Data. 2013.

[36] Mahalli, A.E., Adoption and Barriers to Adoption of Electronic Health Records by Nurses in Three Governmental Hospitals in Eastern Province, Saudi Arabia. Perspectives in health information management, 2015. 12(Fall)

[37] Lorenzi, N.M., et al., How to successfully select and implement electronic health records (EHR) in small ambulatory practice settings. BMC medical informatics and decision making, 2009. 9(1): p. 15

[38] Car, J., et al., The Impact of eHealth on the Quality \& Safety of Healthcare A Systematic Overview \& Synthesis of the Literature. Report for the NHS Connecting for Health Evaluation Programme March $2008 . \quad 2009, \quad 32956$ FFC-BD76-47B7-94D2FFAC56979B74.

[39] Goodman, J.C., L. Gorman, and D. Herrick, Health Information Technology: Benefits and Problems. National Center for Policy Analysis, Washington, 2010.

[40] Sikhondze, N.C. and L. Erasmus, Electronic medical records: a developing and developed country analysis. 2016.

[41] Msiska, K.E.M., A. Kumitawa, and B.J.M.M.J. Kumwenda, Factors affecting the utilisation of electronic medical records system in Malawian central hospitals. 2017. 29(3): p. 247-253.

[42] Shams Amiri, N., Emerge and deploying electronic health records in developing countries. 2012.

[43] Alkraiji, A., T. Jackson, and I.J.C.-W.I.S. Murray, Health data standards and adoption process: Preliminary findings of a qualitative study in Saudi Arabia. 2011. 28(5): p. 345-359.
[44] Eldin, A.S., et al., Evaluation of electronic health records adoption in Egypt. 2013. 3(1): p. 1131-1134.

[45] Stadelmann, J., Assessing readiness for e-health in Egypt: a case study of Cairo University hospitals. 2012.

[46] Baxter, C., R. Dell, and R. Race, Assessing and improving EHR data quality. Journal of AHIMA, 2007. 78(3): p. $69-72$

[47] Arrowood, D., et al., Integrity of the healthcare record. Best practices for EHR documentation. Journal of AHIMA/American Health Information Management Association, 2013. 84(8): p. 58.

[48] Chan, K.S., J.B. Fowles, and J.P. Weiner, Review: electronic health records and the reliability and validity of quality measures: a review of the literature. Medical Care Research and Review, 2010. 67(5): p. 503-527.

[49] Bowman, S., Impact of Electronic Health Record Systems on Information Integrity. 2012.

[50] Darko-Yawson, S. and G. Ellingsen, Assessing and Improving EHRs Data Quality through a Socio-technical Approach. Procedia Computer Science, 2016. 98: p. 243 250.

[51] Kottmann, J., et al., Apache opennlp. 2011

[52] Bayley, K.B., et al., Challenges in using electronic health record data for CER: experience of 4 learning organizations and solutions applied. Medical care, 2013. 51: p. S80-S86.

[53] [Capurro, D., et al., Availability of Structured and Unstructured Clinical Data for Comparative Effectiveness Research and Quality Improvement: A Multi-Site Assessment. eGEMs (Generating Evidence \& Methods to improve patient outcomes). 2014; 2: 1. 2014 , Article.

[54] Dullabh, P.M., et al., How patients can improve the accuracy of their medical records. eGEMs, 2014. 2(3). 